\title{
Critical behavior of the bulk viscosity in QCD
}

\author{
M. Martinez, ${ }^{1}$ T. Schäfer®, ${ }^{1}$ and V. Skokov $\circledast^{1,2}$ \\ ${ }^{1}$ Department of Physics, North Carolina State University, Raleigh, North Carolina 27695, USA \\ ${ }^{2}$ RIKEN-BNL Research Center, Brookhaven National Laboratory, Upton, New York 11973, USA
}

(Received 16 July 2019; published 15 October 2019)

\begin{abstract}
We study the behavior of the bulk viscosity $\zeta$ in QCD near a possible critical end point. We verify the expectation that $(\zeta / s) \sim a\left(\xi / \xi_{0}\right)^{x_{\zeta}}$, where $s$ is the entropy density, $\xi$ is the correlation length, $\xi_{0}$ is the noncritical correlation length, $a$ is a constant, and $x_{\zeta} \simeq 3$. Using a recently developed equation of state that includes a critical point in the universality class of the Ising model we estimate the constant of proportionality $a$. We find that $a$ is typically quite small, $a \sim O\left(10^{-4}\right)$. We observe, however, that this result is sensitive to the commonly made assumption that the Ising temperature axis is approximately aligned with the QCD chemical potential axis. If this is not the case, then the critical $\zeta / s$ can approach the noncritical value of $\eta / s$, where $\eta$ is the shear viscosity, even if the enhancement of the correlation length is modest, $\xi / \xi_{0} \sim 2$.
\end{abstract}

DOI: 10.1103/PhysRevD.100.074017

\section{INTRODUCTION}

There are several programs dedicated to exploring the phase diagram of quantum chromodynamics (QCD) at heavy ion accelerator laboratories around the world [1]. A central feature of the phase diagram is a possible critical end point of a first order phase transition between the hadronic phase and the quark gluon plasma phase. Experimentally, a critical point is expected to manifest itself in terms of a nontrivial beasundm energy, rapidity, or system size dependence of fluctuation observables [2].

In a static system fluctuation observables are controlled by the critical equation of state [3-5]. The equation of state near the QCD critical point is expected to be in the liquidgas (Ising) universality class. The equation of state of the Ising model is known from lattice simulations [6], and accurate parametrizations are available in the literature [7]. More recently, there have been efforts to map the Ising equation of state onto the QCD phase diagram, taking into account information from lattice QCD about the equation of state and the susceptibilities at zero baryon chemical potential $[8,9]$.

The dynamic behavior of fluctuations is expected to be governed by model $\mathrm{H}$ in the classification of dynamical critical phenomena by Hohenberg and Halperin [10-12]. Model $\mathrm{H}$ is a hydrodynamic theory that describes coupling of the order parameter field to a conserved momentum

Published by the American Physical Society under the terms of the Creative Commons Attribution 4.0 International license. Further distribution of this work must maintain attribution to the author(s) and the published article's title, journal citation, and DOI. Funded by SCOAP . density. It predicts the dynamic critical exponent for the relaxation of the order parameter, and the critical behavior of the transport coefficients, the shear viscosity $\eta$, the bulk viscosity $\zeta$, and the thermal conductivity $\kappa$. In model $\mathrm{H}$ fluctuations of the order parameter with wave number $q \sim \xi^{-1}$, where $\xi$ is the correlation length, relax on a timescale $\tau \sim \xi^{z}$, where $z \simeq 3$. This behavior is intermediate between ordinary diffusion $(z \simeq 2)$ and critical relaxation of a conserved charge not coupled to fluctuations of the fluid velocity $(z \simeq 4)$. There is a very mild divergence in the shear viscosity, as well as more pronounced critical behavior in the thermal conductivity and bulk viscosity [10,13-17],

$$
\eta \sim \xi^{0.05}, \quad \kappa \sim \xi^{0.9}, \quad \zeta \sim \xi^{2.8} .
$$

Physical effects related to critical transport phenomena have been observed in ordinary fluids. For example, the critical behavior of the bulk viscosity manifests itself in sound attenuation near the critical end point [17].

Recently, a number of authors have investigated the dynamic evolution of fluctuations in an expanding QCD medium. This includes studies of noncritical correlation functions [18-20], simulations of critical stochastic diffusion in an expanding medium [21], and deterministic frameworks for the evolution of two-point [22-24] or higher $n$-point functions [25] near a critical point.

There is a general expectation that the large critical exponent in Eq. (1), combined with the strong deviation of the QCD equation of state from scale invariance, will lead to a substantial enhancement of the bulk viscosity and to large effects on the evolution of a heavy ion collision near a critical end point $[26,27]$. Our goal in the present work is to study this problem more quantitatively, based on the 
equation of state constructed by Parotto et al. [9]. We will verify the expected scaling behavior and estimate the overall coefficient. We will also study the relaxation of the bulk pressure near a QCD critical point. These results complement earlier studies of noncritical contributions to the bulk viscosity in QCD [28-31].

\section{FLUCTUATIONS OF THE ENERGY DENSITY AND PRESSURE}

Fluctuations of the order parameter are governed by an entropy functional $S=\int d^{3} x s$. The entropy functional of the Ising model is a function of the densities

$$
x^{A}=(\epsilon, \psi),
$$

where $\epsilon$ and $\psi$ are the Ising energy density and order parameter. The corresponding intensive variables are the reduced temperature $r$ and the magnetic field $h$,

$$
X_{A}=-\frac{\partial s}{\partial x^{A}}=(r, h) .
$$

We are following the notation of Landau and Lifshitz [32] as well as Akamatsu et al. [23]. The analogous canonical pair in QCD is

$$
x^{a}=(e, n), \quad X_{a}=(-\beta, \beta \mu),
$$

where $(e, n)$ are the energy and baryon density, $\beta=1 / T$ is the inverse temperature, and $\mu$ is the baryon chemical potential. We will assume that there is a map between the intensive variables in QCD and the corresponding Ising variables; see Fig. 1 and Sec. III. Fluctuations of the QCD pressure are given by

$\delta P=\left.\frac{\partial P}{\partial \beta}\right|_{\beta \mu} \delta \beta+\left.\frac{\partial P}{\partial \beta \mu}\right|_{\beta} \delta(\beta \mu)=-\frac{e+P}{\beta} \delta \beta+\frac{n}{\beta} \delta(\beta \mu)$.

Using the fact that $(\beta, \beta \mu)$ is conjugate to $(e, n)$ we obtain

$$
\delta P=\frac{e+P}{\beta} \frac{\partial s}{\partial(\delta e)}-\frac{n}{\beta} \frac{\partial s}{\partial(\delta n)} .
$$

The map between $(r, h)$ and $(\beta, \beta \mu)$ induces a map between the QCD densities $(e, n)$ on the Ising densities $(\epsilon, \psi)$. We assume that the singular part of the QCD entropy density is proportional to the Ising entropy density, $s^{\operatorname{sing}}(e, n)=$ $A s^{\mathrm{IS}}(\epsilon(e, n), \psi(e, n))$. A common assumption is that the images of the $r$ and $h$ axes in the QCD phase diagram are approximately orthogonal, and that the Ising temperature axis is almost aligned with the QCD chemical potential axis, ${ }^{1} \partial \epsilon / \partial e \simeq 0[8,9,33]$. This implies that

\footnotetext{
${ }^{1}$ We will reconsider this assumption in Sec. V.
}

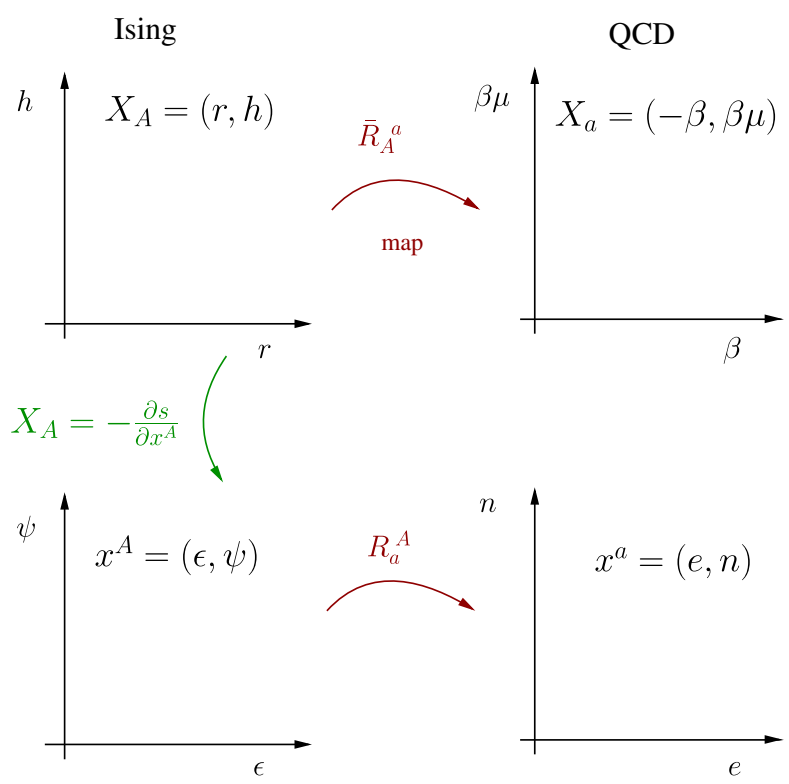

FIG. 1. Map from the Ising model to QCD for intensive variables and conserved densities. Thermodynamic variables are defined in the text. The maps $\bar{R}_{A}^{b}$ and $R_{b}^{A}$ are defined in Eqs. (25) and (26).

$$
\delta P=\frac{e+P}{\beta} \frac{\partial \psi}{\partial(\delta e)} \frac{\partial s^{\mathrm{Is}}}{\partial \psi}-\frac{n}{\beta} \frac{\partial \epsilon}{\partial(\delta n)} \frac{\partial s^{\mathrm{Is}}}{\partial \epsilon} .
$$

Onuki observed that the Ising entropy functional contains a trilinear coupling between $\epsilon$ and $\psi^{2}$, and as a result fluctuations of the pressure couple to $\psi^{2}[16,17]$. This means that correlation functions of $\delta P$ are controlled by the order parameter relaxation rate, and the slow relaxation of $\psi$ leads to a large enhancement in the bulk viscosity.

The presence of a trilinear coupling between $\epsilon$ and $\psi^{2}$ in the Ising entropy $S[\psi, \epsilon]$ can be understood from the relation between entropy and Gibbs free energy ${ }^{2}[34]$

$$
\begin{aligned}
\exp (-\beta G[\psi, r]) & =\int \mathcal{D} \epsilon \exp (S[\psi, \epsilon]-E / T), \\
E & =\int d^{3} x\left(\epsilon+\epsilon_{0}\right),
\end{aligned}
$$

where $E$ is the total energy, $\epsilon_{0}$ is the background energy density, and $T$ is the temperature. For small $\epsilon$ and $\psi$ we can expand the entropy functional as

$$
\begin{aligned}
S[\psi, \epsilon]= & -\int d^{3} x\left\{k(\nabla \psi)^{2}+\frac{v}{2} \psi^{2}+\frac{u}{4} \psi^{4}+\gamma \epsilon \psi^{2}+\frac{1}{2 C_{0}} \epsilon^{2}\right\} \\
& +S_{0}+\frac{E}{T_{0}},
\end{aligned}
$$

\footnotetext{
${ }^{2}$ Note that there is some disagreement in the literature on whether $G[\psi, r]$ should be called free energy or Gibbs free energy. Zinn-Justin refers to this functional as a free energy, and Eq. (3) of Asakawa et al. [8] as well as Parotto et al. [9] follow that notation; but Akamatsu et al. [23] uses the term Gibbs free energy and reserves the term free energy for $F[h, r]$.
} 
where $k, v, u$, and $\gamma$ are constants. $C_{0}$ is the heat capacity, and $S_{0}$ and $T_{0}$ are the background entropy and temperature. The free energy functional is

$$
\beta G[\psi, r]=\int d^{3} x\left\{k(\nabla \psi)^{2}+\frac{\tilde{v}}{2} \psi^{2}+\frac{\tilde{u}}{4} \psi^{4}\right\}
$$

and $\tilde{v}=v+2 \gamma C_{0}\left(T-T_{0}\right) / T_{0}^{2}$, so the Legendre transform generates the $T$ dependence of the correlation length. Note that in this Gaussian approximation $\gamma$ is a constant, independent of $T$. We will see in the following section that, in general, $\gamma$ scales with $T-T_{0}$ according to a nontrivial critical exponent.

Finally, combining Eqs. (7) and (9) we obtain the slow mode contribution to fluctuations of the pressure

$$
\delta P=n T A a_{n \epsilon} \gamma \psi^{2},
$$

where we have defined $a_{n \epsilon}=(\partial \epsilon) /(\partial(\delta n))$.

\section{CRITICAL ENTROPY FUNCTIONAL}

We determine the critical entropy density using the Ising equation of state constructed by Zinn-Justin [7]. We write the Gibbs free energy as [7,35]

$$
G[\psi, r]=h_{0} M_{0} R^{2-\alpha} g(\theta),
$$

where $r=\left(T-T_{c}\right) / T_{c}$. The constants $h_{0}$ and $M_{0}$ will be specified below, $\alpha=0.11$ is the specific heat exponent, and $g(\theta)$ is a function that is given in Appendix. We use the $(R, \theta)$ coordinates

$$
\begin{gathered}
\psi=M_{0} R^{\beta} \theta, \\
r=R\left(1-\theta^{2}\right),
\end{gathered}
$$

where $\beta=0.33$ is the order parameter exponent. Here, $R \in[0, \infty)$ and $\theta \in\left[-\theta_{0}, \theta_{0}\right]$, where $\theta_{0}$ is determined by the condition $\tilde{h}\left(\theta_{0}\right)=0$. Furthermore, $\tilde{h}(\theta)$ is a function that appears in the magnetic equation of state

$$
h=h_{0} R^{\beta \delta} \tilde{h}(\theta),
$$

$\delta=4.78$ is the external field exponent, and $\tilde{h}(\theta)$ is also specified in Appendix. For this parametrization we find $\theta_{0}=1.154$. The value of $\theta_{0}$ determines the boundaries of the first order region for $r<0, \psi_{ \pm}= \pm M_{0} R^{\beta} \theta_{0}$.

We can expand the Gibbs free energy for small $r$ and $\psi$. We find

$G[\psi, r] \simeq h_{0} M_{0}\left\{g_{ \pm} r^{2-\alpha}+\frac{1}{2} m_{ \pm}^{2} r^{2-\alpha-2 \beta}\left(\psi-\psi_{0}\right)^{2}+\cdots\right\}$, where

$$
\begin{aligned}
& g_{ \pm}=\left\{\begin{array}{ll}
-0.84 & r>0 \\
-1.58 & r<0
\end{array},\right. \\
& \psi_{0}=\left\{\begin{array}{cc}
0 & r>0 \\
\psi_{ \pm} & r<0
\end{array} .\right.
\end{aligned}
$$

Note that in the mean field approximation we have $\alpha=0$ and $\beta=1 / 2$, so that $G \sim \frac{1}{2} r \psi^{2}$, as expected. Also note that $2-\alpha-2 \beta=\tilde{\gamma}$ is the susceptibility exponent. The Ising energy density is

$$
\begin{aligned}
\epsilon= & \frac{\partial G}{\partial r} \simeq h_{0} M_{0}\left\{g_{ \pm}(2-\alpha) r^{1-\alpha}+\frac{1}{2} m_{ \pm}^{2}(2-\alpha-2 \beta) r^{1-\alpha-2 \beta}\right. \\
& \left.\times\left(\psi-\psi_{0}\right)^{2}+\cdots\right\} .
\end{aligned}
$$

We can now determine an entropy functional that describes fluctuations of the energy density at constant $\psi$ by performing a Legendre transformation, $s[\psi, \epsilon]=G[\psi, r]-r \epsilon$. Expanding $s$ for small values of the arguments gives

$s[\psi, \epsilon] \simeq\left(-g_{ \pm}\right)(1-\alpha) \mathcal{E}^{\frac{2-\alpha}{1-\alpha}}+\frac{1}{2} m_{ \pm}^{2} \mathcal{E}^{\frac{2-\alpha-2 \beta}{1-\alpha}}\left(\psi-\psi_{0}\right)^{2}+\cdots$,

$$
\mathcal{E}=\frac{\epsilon}{\left(-g_{ \pm}\right)(2-\alpha) h_{0} M_{0}}
$$

We can now read off the trilinear coupling defined in the previous section. We get

$$
\gamma_{ \pm}=\frac{m_{ \pm}^{2}}{2\left(-g_{ \pm}\right)} \frac{2-\alpha-2 \beta}{(2-\alpha)(1-\alpha)}|r|^{1-2 \beta} .
$$

There are two differences compared to the mean field result in the previous section. The first is that the trilinear coupling vanishes near the transition point, with a critical scaling controlled by the exponent ${ }^{3}(1-2 \beta) \simeq 0.34$. We will see that this is more than compensated by the divergence in the order parameter relaxation rate. The second is that there is an amplitude ratio, which means the coupling is different on the first order $(r<0)$ and crossover $(r>0)$ of the transition. We find

$$
\gamma_{ \pm}=\left\{\begin{array}{cc}
0.43 r^{1-2 \beta} & r>0 \\
1.10|r|^{1-2 \beta} & r<0
\end{array}\right.
$$

\footnotetext{
${ }^{3}$ Note that this exponent agrees with the exponent determined using renormalization group arguments [16,34]. Halperin et al. provide a diagrammatic argument that explains why the critical scaling of $\gamma$ is related to the specific heat and susceptibility exponents.
} 
Note that the scaling with $r$ can be converted to a scaling relation involving the correlation length using $|r| \sim \xi^{-1 / \nu}$, with $\nu \simeq 0.63$. Indeed, Zinn-Justin provides a scaling form of the correlation length

$$
\xi=\xi_{0} R^{-\nu} g_{\xi}^{1 / 2}(\theta)
$$

where $\xi_{0}$ is an overall scale and $g_{\xi}(\theta) \simeq\left(1-5 \theta^{2} / 18\right)$ [7].

Parotto et al. construct a map $X_{A}\left(X_{b}\right)$ from intensive QCD variables $X_{a}=(-\beta, \beta \mu)$ to Ising variables $X_{A}=$ $(r, h)$. The specific map considered in [9] is a simple linear relation

$$
\begin{aligned}
\frac{T-T_{c}}{T_{c}} & =\bar{w}\left(r \bar{\rho} \sin \left(\alpha_{1}\right)+h \sin \left(\alpha_{2}\right)\right), \\
\frac{\mu-\mu_{c}}{T_{c}} & =\bar{w}\left(-r \bar{\rho} \cos \left(\alpha_{1}\right)-h \cos \left(\alpha_{2}\right)\right),
\end{aligned}
$$

where $\bar{w}, \bar{\rho}$, and $\alpha_{1,2}$ are parameters. Most of the work in the existing literature $[8,9,33]$ assumes that $\alpha_{1} \simeq 0$ and $\alpha_{2} \simeq \pi / 2$. We will discuss this assumption in more detail below, but for now we will assume that $\alpha_{1}=0$ and $\alpha_{2}=\pi / 2$. Parotto et al. choose $\bar{w}=1, \bar{\rho}=2$, and $A=T_{c}^{3}$. They also use $M_{0}=0.605$ and $h_{0}=0.394$.

To determine the map between the conserved densities we use the fact that the transformation between the intensive variables can be specified in terms of the matrix (see Fig. 1)

$$
\bar{R}_{A}^{b}=\frac{\partial X_{A}}{\partial X_{b}}
$$

This map induces a relationship between the densities $x_{A}\left(x_{b}\right)$, described by

$$
R_{b}^{A}=\frac{\partial x^{A}}{\partial x^{b}}
$$

Since the densities are conjugate to the intensive variables, we must have

$$
R_{b}^{A} \bar{R}_{C}^{b}=\delta_{C}^{A}
$$

which implies that the matrix $R$ is the inverse of $\bar{R}$. In the simple case that $\alpha_{1}=0$ and $\alpha_{2}=\pi / 2$ we obtain

$$
a_{n \epsilon}=\frac{\partial \epsilon}{\partial(\delta n)}=\frac{\bar{\rho} \bar{w}}{A}
$$

where we have used that the normalization of the Ising and QCD entropy functional differ by a factor of $A$.

\section{CRITICAL BULK VISCOSITY}

The bulk viscosity is determined by the Kubo relation

$$
\zeta(\omega)=-\frac{1}{9 \omega} \operatorname{Im} G_{R}^{i i, j j}(\omega, 0)
$$

where $G_{R}^{i j, k l}(\omega, k)$ is the retarded correlation function of the (spatial components) of the stress tensor

$$
\begin{aligned}
& G_{R}^{i j, k l}(\omega, k) \\
& \quad=-i \int d t d^{3} x e^{-i(\omega t-k x)}\left\langle\left[\Pi^{i j}(0,0), \Pi^{k l}(x, t)\right]\right\rangle \theta(t) .
\end{aligned}
$$

The critical behavior arises from the contribution of the nonequilibrium pressures to the trace of the stress tensor, $\frac{1}{3} \Pi^{i i}=\delta P$, with $\delta P$ given in Eq. (11). To compute the righthand side of the Kubo relation Eq. (29) we consider the symmetric correlation function

$$
G_{S}(\omega, k)=c \int d t d^{3} x e^{-i(\omega t-k x)}\left\langle\psi^{2}(0) \psi^{2}(t, x)\right\rangle,
$$

where we have defined $c=\left(n T A a_{n \epsilon} \gamma\right)^{2}$. In statistical field theory we can factorize the correlation function and determine the retarded correlator using the fluctuationdissipation relation. We get

$$
\begin{aligned}
G_{R}(\omega, k=0)= & 2 c \int \frac{d \omega^{\prime}}{2 \pi} \int \frac{d^{3} k}{(2 \pi)^{3}}\left\{\Delta_{R}\left(\omega-\omega^{\prime}, k\right) \Delta_{S}\left(\omega^{\prime}, k\right)\right. \\
& +(S \leftrightarrow R)\},
\end{aligned}
$$

where $\Delta_{S, R}$ are the symmetric and retarded correlation functions of the order parameter $\psi$. The slow dynamics of $\psi$ is governed by a diffusion equation [10]

$$
\frac{\partial \psi}{\partial t}=\lambda_{0} \nabla^{2} \frac{\delta G}{\delta \psi}+\theta
$$

Here, $\lambda_{0}$ is a transport coefficient and $\theta$ is a stochastic force. The noise correlator is

$$
\left\langle\theta(x, t) \theta\left(x^{\prime}, t^{\prime}\right)\right\rangle=-2 \lambda_{0} T \nabla^{2} \delta^{3}\left(x-x^{\prime}\right) \delta\left(t-t^{\prime}\right) .
$$

The diffusion constant is $D=\lambda_{0} / \chi_{0}$ where the susceptibility is given by

$$
\chi_{k}=\frac{\xi^{2}}{1+(k \xi)^{2}} .
$$

The retarded and symmetric order parameter correlation functions are 


$$
\begin{aligned}
& \Delta_{R}(\omega, k)=\chi_{k} \frac{\Gamma_{k}}{-i \omega+\Gamma_{k}}, \\
& \Delta_{S}(\omega, k)=2 \chi_{k} T \frac{\Gamma_{k}}{\omega^{2}+\Gamma_{k}^{2}},
\end{aligned}
$$

where $\Gamma_{k}=\lambda_{0} k^{2} / \chi_{k}$. The bulk viscosity is

$$
\zeta(\omega)=c \int \frac{d^{3} k}{(2 \pi)^{3}} \frac{2 T \chi_{k}^{2}}{-i \omega+2 \Gamma_{k}} .
$$

We note that the order parameter field $\psi$ is dimensionless. We can absorb all dimensionful parameters in Eq. (33) into a length scale, the noncritical correlation length $\xi_{0}$, and a noncritical relaxation time $t_{0}$. These are two free parameters in the model $\mathrm{H}$ description of the QCD critical point, in addition to the parameters that appear in the equation of state. As a rough estimate of $\xi_{0}$ we will use the entropy density of the system and assume that $s \xi_{0}^{3}=1$. We will comment on this choice in Sec. VII. The relaxation time is determined by the diffusion constant, $D_{0}=t_{0} / \xi_{0}^{2}$.

The integral in Eq. (38) is easily performed in the $\omega \rightarrow 0$ limit. We find

$$
\frac{\zeta}{s}=\left(\gamma_{ \pm}^{R} a_{n \epsilon}\right)^{2}\left(t_{0} T\right)^{2}\left(\frac{n}{s}\right)^{2} \frac{3}{32 \pi}\left(\frac{\xi}{\xi_{0}}\right)^{3.92},
$$

where we have taken into account the scaling of $\gamma_{ \pm} \equiv$ $\gamma_{ \pm}^{R} r^{1-2 \beta}$; see Eq. (21). The critical exponent $x_{\zeta}=3.92$ is larger than the one found by Onuki; see Eq. (1). We will show in Sec. V that this is due to the fact that in Eq. (33) we have neglected the coupling of the order parameter to the momentum density of the fluid. The coupling $\gamma_{ \pm}^{R} a_{n e}$ was determined in Eqs. (21) and (28). For typical values of the diffusion constant $t_{0} T$ is of order one; we have used $t_{0} \simeq$ $1.8 \mathrm{fm}$ from [23]. The coefficient $3 /(32 \pi) \sim 3 \times 10^{-2}$ is a loop factor. The main uncertainty in Eq. (39) is the factor $n / s$. For typical equations of state that put the critical end point within reach of the RHIC beam energy scan program this coefficient is quite small. For example, the equation of state shown in Fig. 6 of Parotto et al. [9] has $n / s \simeq 1 / 25$. We then get

$$
\frac{\zeta}{s}=\left(\frac{\xi}{\xi_{0}}\right)^{3.92}\left\{\begin{array}{ll}
6.6 \times 10^{-5} & r>0 \\
4.2 \times 10^{-4} & r<0
\end{array} .\right.
$$

We conclude that the critical enhancement in the bulk viscosity is not large, unless the correlation length becomes quite large. We will study the growth in the effective correlation length in Sec. VI, and we will consider a possible scenario that overcomes the suppression by $n / s$ in the following section.

\section{REFINEMENTS}

In this section, we will discuss two refinements to the calculation described in the previous sections. The first involves the treatment of the order parameter relaxation rate. In model $\mathrm{H}$ it has been shown that the relaxation rate $\Gamma_{k}$ is governed by the coupling of $\psi$ to the momentum density of the fluid [10]. Consider, for example, the choice $\psi=s / n$. Then the conservation laws generate a coupling of the form $(\partial \psi) /(\partial t)=\vec{\pi} \cdot \vec{\nabla} \psi+\cdots$, where $\vec{\pi}$ is the momentum density of the fluid. The momentum relaxation rate has a very weak divergence, so that it makes sense to approximate that rate by the noncritical shear viscosity $\eta_{0}$ of the fluid. This is known as the Kawasaki approximation. Within this approximation $[10,17,36]$

$$
\Gamma_{k}=\frac{T \xi^{-3}}{6 \pi \eta_{0}} K(k \xi),
$$

where

$$
K(x)=\frac{3}{4}\left[1+x^{2}+\left(x^{3}+x^{-1}\right) \arctan (x)\right] .
$$

We will also use a refined parametrization of the order parameter susceptibility

$$
\chi_{k}=\frac{\chi_{0}}{1+(k \xi)^{2-\eta}}
$$

where $\eta \simeq 0.036$ is the correlation function exponent and $\chi_{0}=\xi_{0}^{2}\left(\xi / \xi_{0}\right)^{2-\eta}$. In this context we will continue to define $\xi_{0}$ by $\left(s \xi_{0}^{3}\right)=1$, but we will take $t_{0}=c \xi_{0}$, where $c$ is the speed of light, and the relaxation timescale is set by the noncritical value of $\eta_{0} / s$. We can now recompute the critical contribution to the bulk viscosity in Eq. (38). We find

$$
\frac{\zeta}{s}=\left(\gamma_{ \pm}^{R} a_{n \epsilon}\right)^{2}\left(t_{0} T\right)\left(\frac{n}{s}\right)^{2} \frac{3}{4 \pi^{2}}\left(4 \pi \frac{\eta_{0}}{s}\right) I_{K}\left(\frac{\xi}{\xi_{0}}\right)^{z-\alpha / \nu},
$$

where

$$
I_{K}=\int d y \frac{y^{2}}{K(y)\left(1+y^{2-\eta}\right)^{2}} \simeq 0.649
$$

is the result of a numerical integral, and we have used the hyperscaling relation $\nu d=2-\alpha=2 \beta+\gamma$, where $d=3$ is the number of spatial dimensions. For the Kawasaki function we have $z=3$, which is close to the result in the $\epsilon$ expansion, $z=3.05$. Using the values of $\alpha$ and $\nu$ quoted above we find $z-\alpha / \nu \simeq 2.8$, which agrees with Onuki's result [16]. For $\eta_{0} / s \simeq 1 /(4 \pi)$ the estimate in Eq. (44) is numerically close to the result given above. We find 


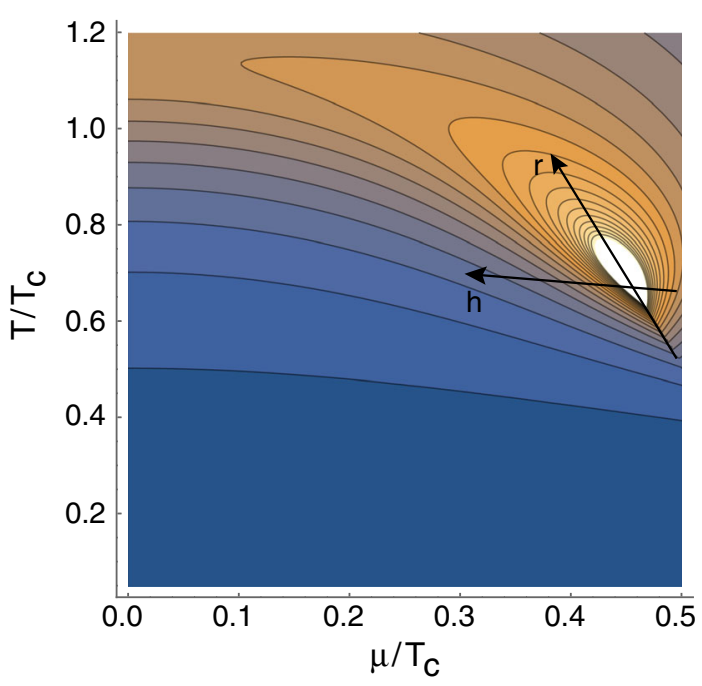

FIG. 2. Ising model axes $r$ and $h$ in a random matrix model of the QCD phase diagram. The axes of the plot are the quark chemical potential $\mu$ and the temperature $T$ in units of the critical temperature in the chiral limit. The parameters of the model were chosen as explained in the text. The contour lines show the chiral susceptibility.

$$
\frac{\zeta}{s}=\left(4 \pi \frac{\eta_{0}}{s}\right)\left(\frac{\xi}{\xi_{0}}\right)^{2.8}\left\{\begin{array}{ll}
8.0 \times 10^{-5} & r>0 \\
5.2 \times 10^{-4} & r<0
\end{array} .\right.
$$

We note that the smallness of the pre-exponent in Eq. (46) is mainly due to the small factor of $(n / s)$. The appearance of this factor is due to the assumption about the orientation of the Ising axes in the QCD phase diagram made above Eq. (7). It was recently observed that close to the chiral limit this assumption is not correct, and that in models of QCD this observation also applies to realistic values of the quark masses [37]. To illustrate this fact we show in Fig. 2 the critical end point and the orientation of the Ising axes in the random matrix model introduced in [38]. The overall scale was fixed by the requirement that $T_{\chi}$, the phase transition temperature in the chiral limit, and $T_{p c}$, the pseudocritical temperature for the physical value of the pion and kaon mass, is the same as in lattice QCD, $T_{\chi} / T_{p c} \simeq\left(132_{-6}^{+3} \mathrm{MeV}\right) /(156.5 \pm 1.5 \mathrm{MeV})$ [39]. In the random matrix model this corresponds to a quark mass $m_{q} \simeq 10 \mathrm{MeV}$. We find $\alpha_{1} \simeq 105^{\circ}$ and $\alpha_{2} \simeq 165^{\circ}$. In this case the dominant contribution to $\delta P$ arises from

$$
\delta P=s T^{2} A a_{e \epsilon} \gamma \psi^{2}
$$

with $a_{e \epsilon}=(\partial \epsilon) /(\partial(\delta e))$. Using the mapping relation in Eqs. (23) and (24) together with Eq. (27) we find $A a_{e \epsilon}=$ $\beta \bar{\rho} \bar{w} \sin \left(\alpha_{1}\right)$ and

$$
\frac{\zeta}{s}=\sin \left(\alpha_{1}\right)^{2}\left(4 \pi \frac{\eta_{0}}{s}\right)\left(\frac{\xi}{\xi_{0}}\right)^{2.8}\left\{\begin{array}{ll}
5.1 \times 10^{-2} & r>0 \\
3.2 \times 10^{-1} & r<0
\end{array} .\right.
$$

With $\sin ^{2}\left(\alpha_{1}\right) \simeq 1 / 4$ from Fig. 2 we observe that, at least on the first order side of the transition, the critical bulk viscosity can reach $\zeta / s \sim 1 /(4 \pi)$, comparable to the noncritical shear viscosity. ${ }^{4}$ Note that Eqs. (46) and (48) are limiting cases, corresponding to $\sin \left(\alpha_{1}\right)^{2} \sim 0$ and $\sin \left(\alpha_{1}\right)^{2} \gtrsim(n / s)^{2}$, of a more general relation that involves both $a_{n \epsilon}$ and $a_{e \epsilon}$. The more general result follows from the coupling $\delta P=\bar{\rho} \bar{w}\left[s \sin \left(\alpha_{1}\right)+n\left(\cos \left(\alpha_{1}\right)-\beta \mu \sin \left(\alpha_{1}\right)\right)\right]$ $T A \gamma \psi^{2}$.

\section{BULK VISCOSITY IN AN EXPANDING SYSTEM}

In a heavy ion collision the growth of the correlation length is limited by the failure of the evolution to go precisely through the critical point and by the finite time available for the correlation length to reach its equilibrium value $[23,40]$. The recent study [23] concludes that the second effect dominates and that $\xi / \xi_{0}$ is limited by $\xi_{\mathrm{KZ}} / \xi_{0}$ where $\xi_{\mathrm{KZ}}$ is the Kibble-Zurek scale. Akamatsu et al. [23] estimate that $\xi_{\mathrm{KZ}} / \xi_{0} \sim 1.33$, so that the critical bulk viscosity in Eq. (40) does not become large.

There is a second effect that appears even if the correlation length does become large. Critical slowing down also implies that the bulk pressure only relaxes slowly to the equilibrium value $\Delta P \simeq \zeta(\vec{\nabla} \cdot \vec{u})$, where $\vec{u}$ is the fluid velocity [22]. The response $\Delta P(t)$ to a timedependent bulk stress $(\vec{\nabla} \cdot \vec{u})\left(t^{\prime}\right)$ is given by

$$
\Delta P(t)=\int^{t} d t^{\prime} G\left(t-t^{\prime}\right)(\vec{\nabla} \cdot \vec{u})\left(t^{\prime}\right),
$$

where $G(t)$ is the Fourier transform of Eq. (38),

$$
G(t)=c \int \frac{d^{3} k}{(2 \pi)^{3}} 2 T \chi_{k}^{2} \exp \left(-2 \Gamma_{k} t\right) .
$$

In the following, we will use the simple form $\Gamma_{k}=\lambda_{0} k^{2} \chi_{k}^{-1}$. The result is easily generalized to the Kawasaki approximation in Eq. (41). The time integral of $G(t)$ is given by the bulk viscosity $\zeta$, and the asymptotic behavior of $G(t)$ for large $t$ is governed by hydrodynamic tails $G(t) \sim 1 / t^{3 / 2}$ $[18,20,41-46]$. The tail of the critical contribution is

$\frac{G(t)}{s T}=\left(\gamma_{ \pm}^{R} a_{n \epsilon}\right)^{2}\left(t_{0} T\right)\left(\frac{n}{s}\right)^{2} \frac{1}{16 \pi^{3 / 2}}\left(\frac{\xi}{\xi_{0}}\right)^{5.92}\left(\frac{t_{0}}{t}\right)^{3 / 2}$,

which has an even stronger dependence on the correlation length than the static value of the bulk viscosity. We note, however, that the asymptotic behavior only sets in at a parametrically late time, $\left(t / t_{0}\right) \gg\left(\xi / \xi_{0}\right)^{4}$.

\footnotetext{
${ }^{4}$ Note that Parotto et al. [9] use $\alpha_{1}=3.85^{\circ}$, based on the curvature of the freeze-out curve. This corresponds to $\sin \left(\alpha_{1}\right)^{2} \simeq 4 \times 10^{-3}$.
} 


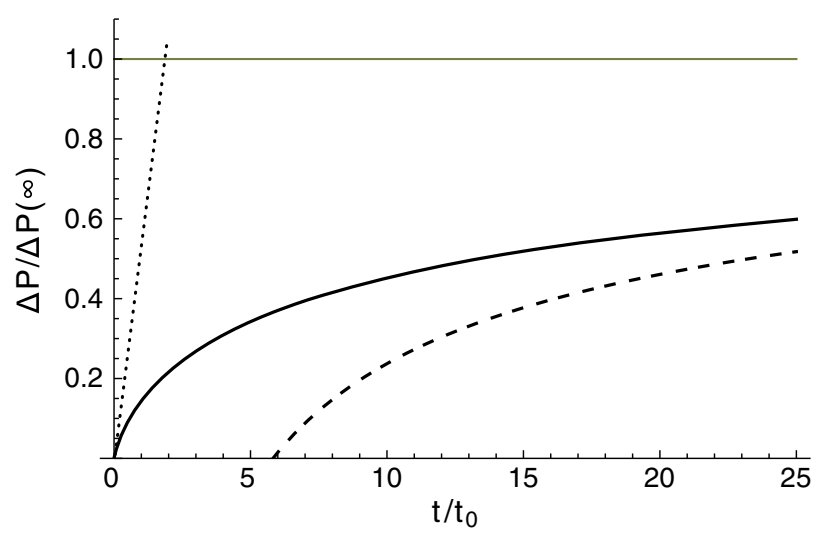

FIG. 3. Relaxation of the bulk pressure $\Delta P(t)$ to its asymptotic value $\Delta P(\infty)$ as a function of $t / t_{0}$, where $t_{0}$ is the noncritical relaxation time. In this figure we have chosen $\xi / \xi_{0}=1.5$. The dotted and dashed lines show the early and late time asymptotics of $\Delta P(t)$.

We may also consider a bulk stress that is turned on at $t=0$ and then study the evolution of $\Delta P(t)$ toward its asymptotic value $\Delta P(\infty)=\zeta(\vec{\nabla} \cdot \vec{u})$. The relaxation is not exponential. Instead, we find very slow relaxation

$$
\Delta P(t) \simeq \Delta P(\infty)\left\{1-\frac{4}{3} \sqrt{\frac{2}{\pi}}\left(\frac{\xi}{\xi_{0}}\right)^{2}\left(\frac{t_{0}}{t}\right)^{1 / 2}\right\}
$$

where, again, the asymptotic behavior requires $t \gg \xi^{4}$. For small $t$ we find that $\Delta P$ grows linearly in $t$,

$$
\Delta P(t)=\frac{8}{3}\left(\frac{\xi_{0}}{\xi}\right)^{4}\left(\frac{t}{t_{0}}\right) \Delta P(\infty)
$$

but the linear growth involves a much smaller power of the correlation length. This behavior is shown in Fig. 3. We observe that even for a modest enhancement of the correlation length, $\xi / \xi_{0}=1.5$, the critical bulk pressure relaxes on a timescale much slower than the natural equilibration time $t_{0}$.

\section{CONCLUSIONS AND OUTLOOK}

In this work we have studied the critical bulk viscosity in QCD. We find that the result is very sensitive to the orientation of the Ising axes in the QCD phase diagram. It is often assumed that the Ising temperature axis is aligned with the QCD chemical potential axis and that the Ising magnetic field axis is orthogonal to that. In that case, the QCD bulk viscosity is suppressed by a factor of $(n / s)^{2}$. If we include a possible misalignment of the Ising axes our final result is given in Eq. (48). We observe that there is significant dependence on what side of the phase transition we are considering; the critical contribution to the bulk viscosity is about an order of magnitude larger on the first order side of the transition. In this regime the critical enhancement of the bulk viscosity may become comparable to the noncritical shear viscosity, even for a modest enhancement of the correlation length, $\xi / \xi_{0} \sim 2$.

We note that the overall scale for $\zeta / s$ is set by the two parameters $\eta_{0} / s$ and $s \xi_{0}^{3}$. While $\eta_{0} / s$ is fairly well constrained by data, this is not the case for $s \xi_{0}^{3}$. We have assumed that $s \xi_{0}^{3} \simeq 1$, based on the idea that in a hadron gas the correlation length cannot be shorter than the distance between particles. However, the exact value of $\xi_{0}$ will depend on the composition of the gas and the precise observable used to define the correlation length. For example, the recent work of Akamatsu et al. [23] uses $\xi_{0} \simeq 1.2 \mathrm{fm}$. Combined with a freeze-out value $s T^{3} \simeq 7$ [47] this number corresponds to $s \xi_{0}^{3} \simeq 5$.

We have also studied the response function for the bulk pressure in the critical regime. As expected, relaxation is very slow. The bulk pressure approaches its equilibrium value as $1 / \sqrt{t}$, and the initial rise of the bulk pressure involves a much smaller power of the correlation length than the scaling of $\zeta$. Note that in the present work we have only considered the Fourier transform of the equilibrium response. A more complete treatment in an expanding system would be based on computing the response from the real time correlation functions of the order parameter in an expanding medium $[20,23]$.

Finally, we may compare our results to simple scaling relations that have been discussed in the literature. Weinberg proposed the relation $\zeta \sim\left(c_{s}^{2}-1 / 3\right)^{2} \eta$, where $c_{s}$ is the speed of sound [48]. This relation is satisfied in the weak coupling limit of QCD [28], and it holds in simple relaxation time models of kinetic theory [31]. Weinberg's relation predicts an enhancement of the bulk viscosity near the critical point, but no critical divergence of the type studied in this work. Based on an analysis of spectral sum rules, Karsch et al. suggested a different scaling relation, $\zeta / s \sim\left(1 / c_{s}^{2}-3\right)$ [26]. This formula predicts a mild divergence of the bulk viscosity, $\zeta / s \sim \xi^{\alpha / \nu}$, much weaker than our result $\zeta / s \sim \xi^{z-\alpha / \nu}$.

Implementations of second order nonconformal relativistic fluid dynamics take into account a bulk viscous relaxation time $\tau_{\zeta}$. A typical assumption, motivated by kinetic theory, is that $\tau_{\zeta} \sim \zeta / P[27,31]$. This relation captures in an approximate way the critical slowing down of the response discussed in Sec. VI, but a simple relaxation time approximation cannot capture the hydrodynamic tail given in Eq. (51).

\section{ACKNOWLEDGMENTS}

We thank M. Stephanov and Yi Yin for useful discussions. This work was supported in part by the U.S. Department of Energy Grant No. DE-FG02-03ER41260 and by the BEST (Beam Energy Scan Theory) DOE Topical Collaboration. 


\section{APPENDIX: ISING EQUATION OF STATE}

We use a parametrization of the Ising equation of state constructed by Zinn-Justin [7]. We write

$$
G[\psi, r]=h_{0} M_{0} R^{2-\alpha} g(\theta)
$$

with

$g(\theta)=g(1)+c_{1}\left(1-\theta^{2}\right)+c_{2}\left(1-\theta^{2}\right)^{2}+c_{3}\left(1-\theta^{2}\right)^{3}$,

and $g(1)=0.0424455$, as well as

$$
c_{1}=0.321329, \quad c_{2}=-1.20375, \quad c_{3}=-0.00126 .
$$

The magnetic equation of state is $h=h_{0} R^{\beta \delta} \tilde{h}(\theta)$ with

$$
\tilde{h}(\theta)=\theta+h_{1} \theta^{3}+h_{2} \theta^{5}
$$

and

$$
h_{1}=0.76201, \quad h_{2}=0.00804
$$

[1] P. Braun-Munzinger, V. Koch, T. Schäfer, and J. Stachel, Properties of hot and dense matter from relativistic heavy ion collisions, Phys. Rep. 621, 76 (2016).

[2] M. A. Stephanov, K. Rajagopal, and E. V. Shuryak, Signatures of the Tricritical Point in QCD, Phys. Rev. Lett. 1998) 4816 ,81).

[3] M. A. Stephanov, Non-Gaussian Fluctuations Near the QCD Critical Point, Phys. Rev. Lett. 102, 032301 (2009).

[4] M. Asakawa, S. Ejiri, and M. Kitazawa, Third Moments of Conserved Charges as Probes of QCD Phase Structure, Phys. Rev. Lett. 103, 262301 (2009).

[5] M. A. Stephanov, On the Sign of Kurtosis Near the QCD Critical Point, Phys. Rev. Lett. 107, 052301 (2011).

[6] J. Engels, L. Fromme, and M. Seniuch, Numerical equation of state from an improved three-dimensional Ising model, Nucl. Phys. B655, 277 (2003).

[7] J. Zinn-Justin, Quantum field theory and critical phenomena, Int. ser. monogr. phys. 113, 1 (2002).

[8] C. Nonaka and M. Asakawa, Hydrodynamical evolution near the QCD critical end point, Phys. Rev. C 71, 044904 (2005).

[9] P. Parotto et al., Lattice-QCD-based equation of state with a critical point, arXiv:1805.05249.

[10] P. C. Hohenberg and B. I. Halperin, Theory of dynamic critical phenomena, Rev. Mod. Phys. 49, 435 (1977).

[11] R. Folk and H. G. Moser, Critical dynamics: A fieldtheoretical approach, J. Phys. A 39, R207 (2006).

[12] D. T. Son and M. A. Stephanov, Dynamic universality class of the QCD critical point, Phys. Rev. D 70, 056001 (2004).

[13] L. Kadanoff and J. Swift, Transport coefficients near the liquid-gas critical point, Phys. Rev. 166, 89 (1968).

[14] D. M. Kroll and J. M. Ruhland, Sound propagation in critical binary mixtures, Phys. Rev. A 23, 371 (1981).

[15] R. A. Ferrell and J. K. Bhattacharjee, Dynamic scaling theory of the critical attenuation and dispersion of sound in a classical fluid: The binary liquid, Phys. Rev. A 31, 1788 (1985).

[16] A. Onuki, Dynamic equations and bulk viscosity near the gas-liquid critical point, Phys. Rev. E 55, 403 (1997).
[17] A. Onuki, Phase Transition Dynamics (Cambridge University Press, Cambridge, England, 2002).

[18] Y. Akamatsu, A. Mazeliauskas, and D. Teaney, A kinetic regime of hydrodynamic fluctuations and long time tails for a Bjorken expansion, Phys. Rev. C 95, 014909 (2017).

[19] Y. Akamatsu, A. Mazeliauskas, and D. Teaney, Bulk viscosity from hydrodynamic fluctuations with relativistic hydrokinetic theory, Phys. Rev. C 97, 024902 (2018).

[20] M. Martinez and T. Schäfer, Stochastic hydrodynamics and long time tails of an expanding conformal charged fluid, Phys. Rev. C 99, 054902 (2019).

[21] M. Nahrgang, M. Bluhm, T. Schäfer, and S. A. Bass, Diffusive dynamics of critical fluctuations near the QCD critical point, Phys. Rev. D 99, 116015 (2019).

[22] M. Stephanov and Y. Yin, Hydrodynamics with parametric slowing down and fluctuations near the critical point, Phys. Rev. D 98, 036006 (2018).

[23] Y. Akamatsu, D. Teaney, F. Yan, and Y. Yin, Transits of the QCD critical point, arXiv:1811.05081.

[24] X. An, G. Basar, M. Stephanov, and H. U. Yee, Relativistic hydrodynamic fluctuations, Phys. Rev. C 100, 024910 (2019).

[25] S. Mukherjee, R. Venugopalan, and Y. Yin, Real time evolution of non-Gaussian cumulants in the QCD critical regime, Phys. Rev. C 92, 034912 (2015).

[26] F. Karsch, D. Kharzeev, and K. Tuchin, Universal properties of bulk viscosity near the QCD phase transition, Phys. Lett. B 663, 217 (2008).

[27] A. Monnai, S. Mukherjee, and Y. Yin, Phenomenological consequences of enhanced bulk viscosity near the QCD critical point, Phys. Rev. C 95, 034902 (2017).

[28] P. B. Arnold, C. Dogan, and G. D. Moore, The bulk viscosity of high-temperature QCD, Phys. Rev. D 74, 085021 (2006).

[29] G. D. Moore and O. Saremi, Bulk viscosity and spectral functions in QCD, J. High Energy Phys. 09 (2008) 015.

[30] E. Lu and G. D. Moore, The bulk viscosity of a pion gas, Phys. Rev. C 83, 044901 (2011). 
[31] K. Dusling and T. Schäfer, Bulk viscosity, particle spectra and flow in heavy-ion collisions, Phys. Rev. C 85, 044909 (2012).

[32] L. D. Landau and E. M. Lifshitz, Statistical Mechanics, Part I, Course of Theoretical Physics Vol. V (Pergamon Press, New York, 1980).

[33] M. Bluhm, M. Nahrgang, S. A. Bass, and T. Schaefer, Impact of resonance decays on critical point signals in netproton fluctuations, Eur. Phys. J. C 77, 210 (2017).

[34] B. I. Halperin, P. C. Hohenberg, and S.-K. Ma, Renormalization group method for critical dynamics: I. Recursion relations and effects of energy conservation, Phys. Rev. B 10, 139 (1974).

[35] P. Schofield, Parametric Representation of the Equation of State Near A Critical Point, Phys. Rev. Lett. 22, 606 (1969).

[36] K. Kawasaki, Kinetic equations and time correlation functions of critical fluctuations, Ann. Phys. (N.Y.) 61, 1 (1970).

[37] M. S. Pradeep and M. Stephanov, Universality of the critical point mapping between Ising model and QCD at small quark mass, Phys. Rev. D 100, 056003 (2019).

[38] A. M. Halasz, A. D. Jackson, R. E. Shrock, M. A. Stephanov, and J. J. M. Verbaarschot, On the phase diagram of QCD, Phys. Rev. D 58, 096007 (1998).

[39] H. T. Ding et al., The Chiral Phase Transition Temperature in $(2+1)$-Flavor QCD, Phys. Rev. Lett. 123, 062002 (2019).
[40] B. Berdnikov and K. Rajagopal, Slowing out-of-equilibrium near the QCD critical point, Phys. Rev. D 61, 105017 (2000).

[41] B. J. Alder and T. E. Wainwright, Velocity Autocorrelations for Hard Spheres, Phys. Rev. Lett. 18, 988 (1967).

[42] M. H. Ernst, E. H. Hauge, and J. M. J. van Leeuwen, Asymptotic time behavior of correlation functions. I. Kinetic terms, Phys. Rev. A 4, 2055 (1971).

[43] Y. Pomeau and P. Résibois, Time dependent correlation functions and mode-mode coupling theories, Phys. Rep. 19, 63 (1975).

[44] P. Kovtun and L. G. Yaffe, Hydrodynamic fluctuations, long time tails, and supersymmetry, Phys. Rev. D 68, 025007 (2003).

[45] P. Kovtun, Lectures on hydrodynamic fluctuations in relativistic theories, J. Phys. A 45, 473001 (2012).

[46] M. Martinez and T. Schäfer, Hydrodynamic tails and a fluctuation bound on the bulk viscosity, Phys. Rev. A 96, 063607 (2017).

[47] J. Cleymans, H. Oeschler, K. Redlich, and S. Wheaton, Comparison of chemical freeze-out criteria in heavy-ion collisions, Phys. Rev. C 73, 034905 (2006).

[48] S. Weinberg, Gravitation and Cosmology (Wiley, New York, 1972). 